Honam Mathematical J. 33 (2011), No. 4, pp. 519-527

http://dx.doi.org/10.5831/HMJ.2011.33.4.519

\title{
A NOTE ON THE WEIGHTED $q$-BERNOULLI NUMBERS AND THE WEIGHTED $q$-BERNSTEIN POLYNOMIALS
}

\author{
D. V. DOLGY AND T. Kim
}

\begin{abstract}
Recently, the modified $q$-Bernoulli numbers and polynomials with weight $\alpha$ are introduced in [3]. In this paper we give some interesting $p$-adic integral representation on $\mathbb{Z}_{p}$ of the weighted $q$-Bernstein polynomials related to the modified $q$-Bernoulli numbers and polynomials with weight $\alpha$. From those integral representation on $\mathbb{Z}_{p}$ of the weighted $q$-Bernstein polynomials, we can derive some identities on the modified $q$-Bernoulli numbers and polynomials with weight $\alpha$.
\end{abstract}

\section{Introduction}

Let $p$ be a fixed prime number. Throughout this paper, $\mathbb{Z}_{p}, \mathbb{Q}_{p}$ and $\mathbb{C}_{p}$ will denote the ring of $p$-adic rational integers, the field of $p$-adic rational numbers, and the completion of algebraic closure of $\mathbb{Q}_{p}$, respectively. Throughout this paper, we assume that $\alpha \in \mathbb{Q}$ and $q \in \mathbb{C}_{p}$ with $\mid 1-$ $\left.q\right|_{p}<p^{-\frac{1}{p-1}}$ so that $q^{x}=\exp (x \log q)$. The $q$-number $[x]_{q}$ is defined by $[x]_{q}=\frac{1-q^{x}}{1-q}$. Let $U D\left(\mathbb{Z}_{p}\right)$ be the space of uniformly differentiable on $\mathbb{Z}_{p}$ and $C\left(\mathbb{Z}_{p}\right)$ be the space of continuous functions on $\mathbb{Z}_{p}$. For $f \in C\left(\mathbb{Z}_{p}\right)$, the weighted $q$-Bernstein operator of order $n$ for $f \in \mathbb{Z}_{p}$ is defined by Kim-Bayad-Kim as follows:

$\mathbb{B}_{n, q}^{(\alpha)}(f)=\sum_{k=0}^{n} f\left(\frac{k}{n}\right)\left(\begin{array}{l}n \\ k\end{array}\right)[x]_{q^{\alpha}}^{k}[1-x]_{q^{-\alpha}}^{n-k}=\sum_{k=0}^{n} f\left(\frac{k}{n}\right) B_{k, n}^{(\alpha)}(x, q), \quad$ see $\left.[1,2]\right)$.

Received September 5, 2011. Accepted September 8, 2011.

2000 Mathematics Subject Classification. 05A30, 11B65, 11B68, 11D88, 11S80.

Key words and phrases. higher order $q$-Bernoulli numbers and polynomials with weight $\alpha, p$-adic $q$-integral. 
Here,

$$
B_{k, n}^{(\alpha)}(x, q)=\left(\begin{array}{l}
n \\
k
\end{array}\right)[x]_{q^{\alpha}}^{k}[1-x]_{q^{-\alpha}}^{n-k}
$$

is called the weighted $q$-Bernstein polynomials of degree $n$ (see $[1,2,7]$ ). Recently, several authors have studied weighted $q$-Bernoulli and Euler numbers (see [1-10]). For $f \in U D\left(\mathbb{Z}_{p}\right)$, the $p$-adic $q$-integral on $\mathbb{Z}_{p}(q$ Volkenborn integral on $\mathbb{Z}_{p}$ ) is defined by Kim as follows (see [4]):

$$
I_{q}(f)=\int_{\mathbb{Z}_{p}} f(x) d \mu_{q}(x)=\lim _{N \rightarrow \infty} \frac{1}{\left[p^{N}\right]_{q}} \sum_{x=0}^{p^{N}-1} f(x) q^{x} .
$$

Recently, Dolgy-Kim-Lee-Rim introduced the modified $q$-Bernoulli numbers with weight $\alpha$ as follows:

$$
\tilde{\beta}_{0, q}^{(\alpha)}=\frac{q-1}{\log q}, \text { and }\left(q^{\alpha} \tilde{\beta}_{q}^{(\alpha)}+1\right)^{n}-\tilde{\beta}_{n, q}^{(\alpha)}= \begin{cases}\frac{\alpha}{[\alpha]_{q}} & \text { if } n=1, \\ 0 & \text { if } n>1\end{cases}
$$

with the usual convention about replacing $\left(\tilde{\beta}_{q}^{(\alpha)}\right)^{n}$ by $\tilde{\beta}_{n, q}^{(\alpha)}$ (see [3,10]). From (3), they also defined the modified $q$-Bernoulli polynomials with weight $\alpha$ as follows:

$$
\begin{aligned}
\tilde{\beta}_{n, q}^{(\alpha)}(x) & =\sum_{l=0}^{n}\left(\begin{array}{l}
n \\
l
\end{array}\right)[x]_{q^{\alpha}}^{n-l} q^{\alpha l x} \tilde{\beta}_{l, q}^{(\alpha)} \\
& =\left([x]_{q^{\alpha}}+q^{\alpha x} \tilde{\beta}_{q}^{(\alpha)}\right)^{n}(\text { see }[3]) .
\end{aligned}
$$

From (3) and (4), we note that

$$
\tilde{\beta}_{n, q}^{(\alpha)}(x)=\frac{\alpha}{(1-q)^{n}[\alpha]_{q}^{n}} \sum_{l=0}^{n}\left(\begin{array}{c}
n \\
l
\end{array}\right)(-1)^{l} q^{\alpha l x} \frac{l}{[\alpha l]_{q}} .
$$

By (2), we easily see that

$$
\int_{\mathbb{Z}_{p}} f(x+n) q^{-x} d \mu_{q}(x)=\int_{\mathbb{Z}_{p}} f(x) q^{-x} d \mu_{q}(x)+\frac{q-1}{\log q} \sum_{l=0}^{n-1} f^{\prime}(l) .
$$

By (3),(4),(5), and (6), we get

$$
\begin{aligned}
\tilde{\beta}_{n, q}^{(\alpha)}(x) & =\int_{\mathbb{Z}_{p}}[x+y]_{q^{\alpha}}^{n} q^{-y} d \mu_{q}(y) \\
& =\frac{\alpha}{(1-q)^{n}[\alpha]_{q}^{n}} \sum_{l=0}^{n}\left(\begin{array}{c}
n \\
l
\end{array}\right)(-1)^{l} q^{\alpha l x} \frac{l}{[\alpha l]_{q}} .
\end{aligned}
$$


The equation (7) is important to derive the main purpose of this paper. In this paper we give a $p$-adic $q$-integral representation on $\mathbb{Z}_{p}$ of the weighted $q$-Bernstein polynomials of order $n$ associated with the modified $q$-Bernoulli numbers and polynomials with weight $\alpha$. From those interval representation on $\mathbb{Z}_{p}$, we derive some interesting identities on the modified $q$-Bernoulli numbers and polynomials with weight $\alpha$.

\section{Identities on the weighted $q$ - Bernstein polynomials and the weighted $q$-Bernoulli numbers.}

For $n, k \in \mathbb{Z}_{+}$, by (1), we get

$$
B_{k, n}^{(\alpha)}(x, q)=B_{n-k, n}^{(\alpha)}\left(1-x, \frac{1}{q}\right) .
$$

From (7), we note that

$$
\begin{aligned}
\tilde{\beta}_{n, q^{-1}}^{(\alpha)}(1-x) & =\int_{\mathbb{Z}_{p}}\left[1-x+x_{1}\right]_{q^{-\alpha}}^{n} q^{x} d \mu_{q^{-1}}\left(x_{1}\right) \\
& =q^{\alpha n-1}(-1)^{n} \tilde{\beta}_{n, q}^{(\alpha)}(x) .
\end{aligned}
$$

By (2), (6), and (7), we see that

$$
\begin{aligned}
\frac{1}{q} \int_{\mathbb{Z}_{p}}[1-x]_{q^{-\alpha}}^{n} q^{-x} d \mu_{q}(x) & =(-1)^{n} q^{\alpha n-1} \int_{\mathbb{Z}_{p}}[x-1]_{q^{\alpha}}^{n} d \mu_{q}(x) \\
& =(-1)^{n} q^{\alpha n-1} \tilde{\beta}_{n, q}^{(\alpha)}(-1) .
\end{aligned}
$$

From (7) and (8), we can derive the following equation:

$$
\frac{1}{q} \int_{\mathbb{Z}_{p}}[1-x]_{q^{-\alpha}}^{n} q^{-x} d \mu_{q}(x)=(-1)^{n} q^{\alpha n-1} \tilde{\beta}_{n, q}^{(\alpha)}(-1)=\tilde{\beta}_{n, q^{-1}}^{(\alpha)}(2) .
$$

By (3) and (4), we get

$$
\begin{aligned}
\tilde{\beta}_{n, q}^{(\alpha)}(2) & =\left(q^{2 \alpha} \tilde{\beta}_{q}^{(\alpha)}+[2]_{q^{\alpha}}\right)^{n}=\left(q^{\alpha}\left(q^{\alpha} \tilde{\beta}_{q}^{(\alpha)}+1\right)+1\right)^{n} \\
& =\sum_{l=0}^{n}\left(\begin{array}{c}
n \\
l
\end{array}\right) q^{\alpha l}\left(q^{\alpha} \tilde{\beta}_{q}^{(\alpha)}+1\right)^{l} \\
& =\tilde{\beta}_{0, q}^{(\alpha)}+n q^{\alpha}\left(\frac{\alpha}{[\alpha]_{q}}+\tilde{\beta}_{1, q}^{(\alpha)}\right)+\sum_{l=2}^{n}\left(\begin{array}{c}
n \\
l
\end{array}\right) q^{\alpha l} \tilde{\beta}_{l, q}^{(\alpha)} \\
& =n q^{\alpha} \frac{\alpha}{[\alpha]_{q}}+\sum_{l=0}^{n}\left(\begin{array}{c}
n \\
l
\end{array}\right) q^{\alpha l} \tilde{\beta}_{l, q}^{(\alpha)} .
\end{aligned}
$$


For $n \in \mathbb{N}$ with $n \geq 2$, by (3) and (11), we get

$$
\tilde{\beta}_{n, q}^{(\alpha)}(2)-n q^{\alpha} \frac{\alpha}{[\alpha]_{q}}=\tilde{\beta}_{n, q}^{(\alpha)}
$$

From (10) and (12), we have

$$
\frac{1}{q} \int_{\mathbb{Z}_{p}}[1-x]_{q^{-\alpha}}^{n} q^{-x} d \mu_{q}(x)=\tilde{\beta}_{n, q^{-1}}^{(\alpha)}(2)=\frac{n}{q} \frac{\alpha}{[\alpha]_{q}}+\tilde{\beta}_{n, q^{-1}}^{(\alpha)} \quad \text { if } n \geq 2
$$

Let us take the $p$-adic $q$-integral on $\mathbb{Z}_{p}$ for one weighted $q$-Bernstein polynomials as follows:

$$
\begin{aligned}
\int_{\mathbb{Z}_{p}} B_{k, n}^{(\alpha)}(x, q) q^{-x} d \mu_{q}(x) & =\left(\begin{array}{l}
n \\
k
\end{array}\right) \int_{\mathbb{Z}_{p}}[x]_{q^{\alpha}}^{k}[1-x]_{q^{-\alpha}}^{n-k} q^{-x} d \mu_{q}(x) \\
& =\left(\begin{array}{l}
n \\
k
\end{array}\right) \sum_{l=0}^{n-k}\left(\begin{array}{c}
n-k \\
l
\end{array}\right)(-1)^{l} \int_{\mathbb{Z}_{p}}[x]_{q^{\alpha}}^{k+l} q^{-x} d \mu_{q}(x) \\
& =\left(\begin{array}{l}
n \\
k
\end{array}\right) \sum_{l=0}^{n-k}\left(\begin{array}{c}
n-k \\
l
\end{array}\right)(-1)^{l} \tilde{\beta}_{k+l, q}^{(\alpha)} .
\end{aligned}
$$

By the symmetry of the weighted $q$-Bernstein polynomials, we get

$$
\begin{aligned}
\int_{\mathbb{Z}_{p}} B_{k, n}^{(\alpha)}(x, q) q^{-x} d \mu_{q}(x) & =\int_{\mathbb{Z}_{p}} B_{n-k, n}^{(\alpha)}\left(1-x, \frac{1}{q}\right) q^{-x} d \mu_{q}(x) \\
& =\left(\begin{array}{l}
n \\
k
\end{array}\right) \sum_{l=0}^{k}\left(\begin{array}{l}
k \\
l
\end{array}\right)(-1)^{k+l} \int_{\mathbb{Z}_{p}}[1-x]_{q^{-\alpha}}^{n-l} q^{-x} d \mu_{q}(x) \\
& =q\left(\begin{array}{l}
n \\
k
\end{array}\right) \sum_{l=0}^{k}\left(\begin{array}{l}
k \\
l
\end{array}\right)(-1)^{k+l}\left(\frac{1}{q} \int_{\mathbb{Z}_{p}}[1-x]_{q^{-\alpha}}^{n-l} q^{-x} d \mu_{q}(x)\right) \\
& =q\left(\begin{array}{l}
n \\
k
\end{array}\right) \sum_{l=0}^{k}\left(\begin{array}{l}
k \\
l
\end{array}\right)(-1)^{k+l}\left(\frac{n-l}{q} \frac{\alpha}{[\alpha]_{q}}+\tilde{\beta}_{n-l, q^{-1}}^{(\alpha)}\right)
\end{aligned}
$$

if $n-k>1$.

Therefore, by (15), we obtain the following theorem. 
Theorem 1. For $n, k \in \mathbb{Z}_{+}$with $n-k>1$, we have

$$
\int_{\mathbb{Z}_{p}} B_{k, n}^{(\alpha)}(x, q) q^{-x} d \mu_{q}(x)= \begin{cases}n \frac{\alpha}{[\alpha]_{q}}+q \tilde{\beta}_{n, q^{-1}}^{(\alpha)} & \text { if } k=0, \\
n\left(\frac{\alpha}{[\alpha]_{q}}-q \tilde{\beta}_{n, q^{-1}}^{(\alpha)}+q \tilde{\beta}_{n-1, q^{-1}}^{(\alpha)}\right) & \text { if } k=1, \\
q\left(\begin{array}{l}
n \\
k
\end{array}\right) \sum_{l=0}^{k}\left(\begin{array}{c}
k \\
l
\end{array}\right)(-1)^{k+l} \tilde{\beta}_{n-l, q^{-1}}^{(\alpha)} & \text { if } k \geq 2 .\end{cases}
$$

From (14) and Theorem 1, we can derive the following corollary.

Corollary 2. For $n, k \in \mathbb{Z}_{+}$with $n-k>1$, we have

$$
\sum_{l=0}^{n-k}\left(\begin{array}{c}
n-k \\
l
\end{array}\right)(-1)^{l} \tilde{\beta}_{k+l, q}^{(\alpha)}=q \sum_{l=0}^{k}\left(\begin{array}{l}
k \\
l
\end{array}\right)(-1)^{k+l} \tilde{\beta}_{n-l, q^{-1}}^{(\alpha)} \quad \text { if } k \geq 2 .
$$

In particular, when $k=0$ and $k=1$, one has

$$
\sum_{l=0}^{n-k}\left(\begin{array}{c}
n-k \\
l
\end{array}\right)(-1)^{l} \tilde{\beta}_{k+l, q}^{(\alpha)}= \begin{cases}n \frac{\alpha}{[\alpha]_{q}}+q \tilde{\beta}_{n, q^{-1}}^{(\alpha)} & \text { if } k=0 \\
\frac{\alpha}{[\alpha]_{q}}-q \tilde{\beta}_{n, q^{-1}}^{(\alpha)}+q \tilde{\beta}_{n-1, q^{-1}}^{(\alpha)} & \text { if } k=1\end{cases}
$$

Let $m, n, k \in \mathbb{Z}_{+}$with $m+n-2 k>1$. Then we get

$$
\begin{aligned}
\int_{\mathbb{Z}_{p}} B_{k, n}^{(\alpha)}(x, q) B_{k, m}^{(\alpha)} & (x, q) q^{-x} d \mu_{q}(x) \\
& =\left(\begin{array}{c}
n \\
k
\end{array}\right)\left(\begin{array}{c}
m \\
k
\end{array}\right) \int_{\mathbb{Z}_{p}}[x]_{q^{\alpha}}^{2 k}[1-x]_{q^{-\alpha}}^{n+m-2 k} q^{-x} d \mu_{q}(x) \\
& =\left(\begin{array}{c}
n \\
k
\end{array}\right)\left(\begin{array}{c}
m \\
k
\end{array}\right) \sum_{l=0}^{2 k}\left(\begin{array}{c}
2 k \\
l
\end{array}\right)(-1)^{l+2 k} \int_{\mathbb{Z}_{p}}[1-x]_{q^{-\alpha}}^{n+m-l} q^{-x} d \mu_{q}(x) \\
& =q\left(\begin{array}{c}
n \\
k
\end{array}\right)\left(\begin{array}{c}
m \\
k
\end{array}\right) \sum_{l=0}^{2 k}\left(\begin{array}{c}
2 k \\
l
\end{array}\right)(-1)^{l+2 k}\left(\frac{1}{q} \int_{\mathbb{Z}_{p}}[1-x]_{q^{-\alpha}}^{n+m-l} q^{-x} d \mu_{q}(x)\right) .
\end{aligned}
$$


By (13) and (16), we get

$$
\begin{aligned}
& \int_{\mathbb{Z}_{p}} B_{k, n}^{(\alpha)}(x, q) B_{k, m}^{(\alpha)}(x, q) q^{-x} d \mu_{q}(x) \\
& =q\left(\begin{array}{l}
n \\
k
\end{array}\right)\left(\begin{array}{l}
m \\
k
\end{array}\right) \sum_{l=0}^{2 k}\left(\begin{array}{c}
2 k \\
l
\end{array}\right)(-1)^{l+2 k}\left(\frac{(n+m-l) \alpha}{q[\alpha]_{q}}+\tilde{\beta}_{n+m-l, q^{-1}}^{(\alpha)}\right) \\
& =\left\{\begin{array}{cl}
(n+m) \frac{\alpha}{[\alpha]_{q}}+q \tilde{\beta}_{n+m, q^{-1}}^{(\alpha)} & \text { if } k=0, \\
q\left(\begin{array}{c}
n \\
k
\end{array}\right)\left(\begin{array}{c}
m \\
k
\end{array}\right) \sum_{l=0}^{2 k}\left(\begin{array}{c}
2 k \\
l
\end{array}\right)(-1)^{l+2 k} \tilde{\beta}_{n+m-l, q^{-1}}^{(\alpha)} & \text { if } k>0 .
\end{array}\right.
\end{aligned}
$$

Therefore, by (17), we obtain the following theorem.

Theorem 3. For $m, n, k \in \mathbb{Z}_{+}$with $m+n-2 k>1$, we have

$$
\begin{gathered}
\int_{\mathbb{Z}_{p}} B_{k, n}^{(\alpha)}(x, q) B_{k, m}^{(\alpha)}(x, q) q^{-x} d \mu_{q}(x) \\
=\left\{\begin{array}{cc}
(n+m) \frac{\alpha}{[\alpha]_{q}}+q \tilde{\beta}_{n+m, q^{-1}}^{(\alpha)} & \text { if } k=0, \\
q\left(\begin{array}{c}
n \\
k
\end{array}\right)\left(\begin{array}{c}
m \\
k
\end{array}\right) \sum_{l=0}^{2 k}\left(\begin{array}{c}
2 k \\
l
\end{array}\right)(-1)^{l+2 k} \tilde{\beta}_{n+m-l, q^{-1}}^{(\alpha)} & \text { if } k>0 .
\end{array}\right.
\end{gathered}
$$

For $m, n, k \in \mathbb{Z}_{+}$, it is easy to show that

$$
\begin{aligned}
& \int_{\mathbb{Z}_{p}} B_{k, n}^{(\alpha)}(x, q) B_{k, m}^{(\alpha)}(x, q) q^{-x} d \mu_{q}(x) \\
& =\left(\begin{array}{l}
n \\
k
\end{array}\right)\left(\begin{array}{c}
m \\
k
\end{array}\right) \int_{\mathbb{Z}_{p}}[x]_{q^{\alpha}}^{2 k}[1-x]_{q^{-\alpha}}^{n+m-2 k} q^{-x} d \mu_{q}(x) \\
& =\left(\begin{array}{l}
n \\
k
\end{array}\right)\left(\begin{array}{c}
m \\
k
\end{array}\right) \sum_{l=0}^{n+m-2 k}\left(\begin{array}{c}
n+m-2 k \\
l
\end{array}\right)(-1)^{l} \\
& \int_{\mathbb{Z}_{p}}[x]_{q^{\alpha}}^{2 k+l} q^{-x} d \mu_{q}(x) \\
& =\left(\begin{array}{l}
n \\
k
\end{array}\right)\left(\begin{array}{l}
m \\
k
\end{array}\right) \sum_{l=0}^{n+m-2 k}\left(\begin{array}{c}
n+m-2 k \\
l
\end{array}\right)(-1)^{l} \tilde{\beta}_{2 k+l, q}^{(\alpha)} .
\end{aligned}
$$

By comparing the coefficients on the both sides of Theorem 3 and (18), we obtain the following corollary. 
Corollary 4. For $m, n, k \in \mathbb{Z}_{+}$with $m+n-2 k>1$, we have

$$
\sum_{l=0}^{n+m}\left(\begin{array}{c}
n+m \\
l
\end{array}\right)(-1)^{l} \tilde{\beta}_{2 k+l, q}^{(\alpha)}=(n+m) \frac{\alpha}{[\alpha]_{q}}+q \tilde{\beta}_{n+m, q^{-1}}^{(\alpha)} .
$$

In particular, if $k>0$, then we get

$$
\sum_{l=0}^{n+m-2 k}\left(\begin{array}{c}
n+m-2 k \\
l
\end{array}\right)(-1)^{l} \tilde{\beta}_{2 k+l, q}^{(\alpha)}=q \sum_{l=0}^{2 k}\left(\begin{array}{c}
2 k \\
l
\end{array}\right)(-1)^{l+2 k} \tilde{\beta}_{n+m-l, q^{-1}}^{(\alpha)} .
$$

By induction hypothesis, we obtain the following theorem.

Theorem 5. For $s \in \mathbb{N}$ and $k, n_{1}, n_{2}, \cdots, n_{s} \in \mathbb{Z}_{+}$with $n_{1}+n_{2}+$ $\cdots+n_{s}-s k>1$, we have

$$
\begin{gathered}
\int_{\mathbb{Z}_{p}}\left(\prod_{i=1}^{s} B_{k, n_{i}}^{(\alpha)}(x, q)\right) q^{-x} d \mu_{q}(x) \\
= \begin{cases}\frac{\left(n_{1}+n_{2}+\cdots+n_{s}\right) \alpha}{[\alpha]_{q}}+q \tilde{\beta}_{n_{1}+n_{2}+\cdots+n_{s}, q^{-1}}^{(\alpha)} & \text { if } k=0, \\
q\left(\prod_{i=1}^{s}\left(\begin{array}{c}
n_{i} \\
k
\end{array}\right)\right) \sum_{l=0}^{s k}\left(\begin{array}{c}
s k \\
l
\end{array}\right)(-1)^{l+s k} \tilde{\beta}_{\sum_{i=1}^{s} n_{i}-l, q^{-1}}^{(\alpha)} & \text { if } k>0 .\end{cases}
\end{gathered}
$$

For $s \in \mathbb{N}$ and $k, n_{1}, n_{2}, \cdots, n_{s} \in \mathbb{Z}_{+}$with $n_{1}+n_{2}+\cdots+n_{s}-s k>1$, By simple calculation, we easily get

$$
\begin{array}{rl}
\int_{\mathbb{Z}_{p}}\left(\prod_{i=1}^{s} B_{k, n_{i}}^{(\alpha)}(x, q)\right) q^{-x} & d \mu_{q}(x) \\
=\left(\prod_{i=1}^{s}\left(\begin{array}{c}
n_{i} \\
k
\end{array}\right)\right) \sum_{l=0}^{n_{1}+\cdots+n_{s}-s k}\left(\begin{array}{c}
n_{1}+\cdots+n_{s}-s k \\
l
\end{array}\right)(-1)^{l} \tilde{\beta}_{l+s k, q}^{(\alpha)} .
\end{array}
$$

By comparing the coefficients on the both sides of Theorem 5 and (19), we obtain the following corollary.

Corollary 6. For $s \in \mathbb{N}$ and $k, n_{1}, \cdots, n_{s} \in \mathbb{Z}_{+}$with $n_{1}+\cdots+n_{s}-$ $s k>1$, we have

$$
\begin{gathered}
q \sum_{l=0}^{s k}\left(\begin{array}{c}
s k \\
l
\end{array}\right)(-1)^{l+s k} \tilde{\beta}_{n_{1}+\cdots+n_{s}-l, q^{-1}}^{(\alpha)} \\
=\sum_{l=0}^{\sum_{i=1}^{s} n_{i}-s k}\left(\begin{array}{c}
\sum_{i=1}^{s} n_{i}-s k \\
l
\end{array}\right)(-1)^{l} \tilde{\beta}_{l+s k, q}^{(\alpha)} \text { if } k>0 .
\end{gathered}
$$


In particular, $k=0$, we get

$$
\sum_{l=0}^{\sum_{i=1}^{s} n_{i}}\left(\begin{array}{c}
\sum_{i=1}^{s} n_{i} \\
l
\end{array}\right)(-1)^{l} \tilde{\beta}_{l, q}^{(\alpha)}=\frac{\left(n_{1}+n_{2}+\cdots+n_{s}\right) \alpha}{[\alpha]_{q}}+q \tilde{\beta}_{n_{1}+n_{2}+\cdots+n_{s}, q^{-1}}^{(\alpha)} .
$$

\section{References}

[1] S. Araci, D. Erdal, J. J. Seo, A study on the fermionic p-adic q-integral on $\mathbb{Z}_{p}$ associated with weighted $q$-Bernstein and q-Genocchi polynomials, Abstract and Applied Analysis 2011(2011), Article in Press http:www.hindawi.com./26592680.

[2] T. Kim, A. Bayad, Y.-H. Kim, A study on the p-adic q-integral on $\mathbb{Z}_{p}$ associated with the weighted $q$-Bernstein and $q$-Bernoulli polynomials, J. Ineq. Appl. 2011(2011), Article ID 29513821, 8 pages.

[3] D. V. Dolgy, T. Kim, S. H. Lee, B. Lee, S. H. Rim, A note on the modified $q$-Bernoulli numbers and polynomials with weight $\alpha$ (communicated).

[4] T. Kim, q-Volkenborn integration, Russ. J. Math. Phys. 9 (2002), 288-299.

[5] T. Kim, A note on q-Bernstein polynomials, Russ. J. Math. Phys. 18 (2011), $73-82$.

[6] T. Kim, B. Lee, J. Choi, Y. H. Kim, Y. H. Kim, S, H. Rim, On the q-Euler numbers and weighted q-Bernstein polynomials, Adv. Stud. Contemp. Math. 21 (2011), 13-18.

[7] L. C. Jang, A family of Barnes-type multiple twisted q-Euler numbers and polynomials related to Fermionic p-adic invariant integrals on $\mathbb{Z}_{p}$, J. Comput. Anal. Appl. 13 (2011), 376-387.

[8] Y. Simsek, Special functions related to Dedekind-type DC-sums and their applications, Russ. J. Math. Phys. 17 (2010), 495-508.

[9] S. H. Rim, E.-J. Moon, S.-J. Lee, J.-H. Jin, On the q-Genocchi numbers and polynomials associated with q-zeta function, Proc. Jangjeon Math. Soc. 12 (2009), 261-267.

[10] T. Kim, S.-H. Lee, D. V. Dolgy, C. S. Ryoo A note on the generalized $q$-Bernoulli measures with weight $\alpha$, Abstract and Applied Analysis. 2011 (2011), Article ID 867217, 9 pages.

D. V. Dolgy

Institute of Mathematics and Computer Sciences, Far Eastern Federal University,

Vladivostok 690060, Russia.

E-mail: d_dol@mail.ru 
T. Kim

Division of General Education-Mathematics, Kwangwoon University, Seoul 139-701, Republic of Korea.

E-mail: tkkim@kw.ac.kr 